\title{
Enfermedad gingival asociada al paciente pediátrico asmático
}

Indhira Del Valle Carfi Marin ${ }^{1}$ Marco Alarcón Palacios ${ }^{2}$

${ }^{1}$ Residente del Programa de Especialización en Periodoncia e implantes.

2Docente del Departamento Académico de Clínica Estomatológica.

Facultad de Estomatología. Universidad Peruana Cayetano Heredia.

\section{Correspondencia}

Indhira Del Valle Carfi Marin

Honorio Delgado 430 - Lima 31, Perú.

e-mail: indhira.carfi.m@upch.pe

Recibido : 6 de junio de 2011

Aceptado : 10 de noviembre de 2011

\section{Introducción}

La Organización Mundial de la Salud (OMS) en 1994, a través de un consenso internacional, intentó definir calidad de vida como la percepción personal de un individuo de su situación en la vida, dentro del contexto cultural y de los valores en que vive y reacción con sus objetivos, expectativas y valores e intereses, afirmando que la calidad de vida (CV) no es igual a estado de salud, estilo de vida, satisfacción con la vida, estado mental ni bienestar, sino que es un concepto multidimensional que debe tener en cuenta la percepción por parte del individuo de éste y otros conceptos de la vida (1).

El asma es la enfermedad crónica más frecuente entre los niños y constituye un grave problema de salud pública. Varios estudios han demostrado una mayor prevalencia de asma, así como su morbilidad y mortalidad en varios países en las últimas décadas. En un estudio realizado en América Latina, la prevalencia de asma en los niños de 6-7 años varió desde 4,1 hasta 26,9\%, y las sibilancias en los últimos 12

Carfi-Marín I, Alarcón-Palacios M. Enfermedad gingival asociada al paciente pediátrico asmático. Rev Estomatol Herediana. 2011; 21(4):237-239.

\section{RESUMEN}

El asma es una enfermedad inflamatoria crónica del sistema respiratorio, caracterizada por ser hipersensible y episódica, reversible y con síntomas de abstracción del flujo de aire. La prevalencia del asma ha ido en aumento a través de todas las edades, sexo y grupos raciales; y resulta ser mayor entre los niños que en adultos. Últimamente ha sido asociada en numerosas investigaciones a la prevalencia de caries y enfermedad periodontal, donde diversos factores y la respuesta inmune actúan de manera similar en ambas patologías. Esta población infantil requiere de atención especial en cuanto al tratamiento y la medicación utilizada.

Palabras clave: ASMA / OBSTRUCCIÓN DE LAS VÍAS AÉREAS / DISNEA / GINGIVITIS.

Gingival disease associated with pediatric asthma patient

ABSTRACT

Asthma is a chronic inflammatory disease of the respiratory system, characterized by being hypersensitive and episodic and having reversible symptoms of airflow abstraction. Asthma prevalence has increased across all ages, sex and racial groups and is higher among children than adults. Lately it has been associated with dental caries and periodontal disease, where different factors and the immune response acts similarly in both conditions. This population requires special attention on treatment and medication used.

Key words: ASTHMA / AIRWAY OBSTRUCTION / AIRWAY OBSTRUCTION / DYSPNEA / GINGIVITIS.

meses, 8,6 a $32,1 \%$ (2).

El asma infantil es una enfermedad multifactorial, habiendo sido asociados a factores genéticos, ambientales, de la gesta Socioeconómicos y otros, en varios estudios en niños y adolescentes. Cuestionarios estandarizados han sido utilizados como principal instrumento para medir la prevalencia de asma en la población. El Estudio Internacional de Asma y Alergias en la infancia (ISAAC) es una iniciativa para establecer la metodología normalizada para facilitar las comparaciones internacionales, generar nuevas hipótesis e investigar las ya existentes. El asma, sin duda, causa un profundo impacto en los niños, sus familias y la sociedad (2).

\section{Asma}

El asma es una enfermedad crónica que se caracteriza por ataques recurrentes de disnea y sibilancias, que varían en severidad y frecuencia de una persona a otra. Los síntomas pueden sobrevenir varias veces al día o a la semana, y en algunas personas se agravan durante la actividad física o por la noche (3).
También se define como un trastorno inflamatorio crónico de las vías respiratorias, caracterizada por episodios y síntomas reversibles de la obstrucción del flujo aéreo (4).

El asma es un problema creciente de salud pública que afectan a más de 300 millones de personas en todo el mundo. Se estima que un adicional de 100 millones puede ser diagnosticado con el asma en el año 2025. Además, se ha convertido en una de las afecciones más comunes durante la infancia y ha ido aumentando en todo el mundo durante los últimos años $(5,6)$.

La mayoría de los casos de asma se dan en países en desarrollo. Los niños tienden a desarrollar más ataques de asma que las niñas. La educación de los padres puede reducir los factores que desencadenan los episodios de asma. El uso de medidas preventivas ayuda a reducir la mortalidad (6).

Recientemente comparaciones internacionales de prevalencia de asma han permitido entender esta distribución a nivel mundial. Mientras Europa occidental tiene alta prevalencia de asma a nivel 
mundial (ECRHS), el estudio internacional de asma y alergias en niños (ISAAC) encontró que la prevalencia de sibilancias son particularmente altas en los países de habla inglesa y en algunos países de América Latina, como Perú y Costa Rica (6).

Los niveles actuales de control del asma en latinoamérica están muy por debajo de las metas establecidas para el manejo del asma a largo plazo. En general, sólo el 2,4\% de los pacientes cumplen con todos los criterios para el total control del asma (7). Un estudio en escolares chilenos demostró prevalencias de asma bronquial de 9,7 a $16,5 \%$ en el grupo de 6 y 7 años y 7,3 a 12,4\% para adolescentes entre 13 a 14 años8; y otro estudio realizado en escolares de algunas ciudades de Brasil encontraron una prevalencia acumulativa diagnostica del asma fue de 7,3\% para los niños y 4,9\% para niñas de 6-7 años; y 9,8 y $10,2 \%$ respectivamente, para los de 13-14 años (2).

Dentro de los signos y síntomas de la enfermedad encontramos los siguientes:

- Disnea: episódica o variable.

- Sibilancias.

- Tos: nocturna, seca e irritativa.

- Opresión torácica.

- Periodos asintomáticos.

En cuanto a diagnósticos diferenciales:

- EPOC.

- Laringitis, traqueitis y bronquitis.

- Bronquiectasias.

- Insuficiencia cardiaca congestiva.

- Cáncer de pulmón, laríngeo.

- Toses psicógenas.

De la misma manera, existen agravantes que influyen en el desarrollo o activación de la enfermedad como son: el polvo doméstico, animales, salidas al campo, humo de tabaco, ambientes cargados, cambios estacionales, productos químicos irritantes, emociones extremas, medicamentos: aspirina, beta bloqueadores, AINES (9).

El 10\% de niños en época preescolar se ven afectados por los síntomas del asma, mientras que la cifra de niños menores de 2 años es de aproximadamente $20 \%$; teniendo los niños preescolares con asma una mayor incidencia de caries, una mayor prevalencia de gingivitis y un mayor consumo de bebidas azucaradas. Niños en edad preescolar con asma requieren una atención especial de médicos y odontólogos, diseñando programas preventivos de forma individual (10).

Los informes anteriores indican que la etiología y gravedad del asma se basa en una compleja interacción entre el medio ambiente, el ámbito social, y los factores genéticos, incluyendo la edad, la etnia, la migración, género, lugar de residencia y status socio-económico. Por otra parte, Se ha encontrado que influyen en el estado socio-demográfico de la susceptibilidad a los alérgenos, la prevalencia del asma y las hospitalizaciones por asma. Estos hallazgos pueden explicar la alta prevalencia $(17,2 \%)$ del asma en la población actual (11).

Martínez et al. (12) encontraron que los síntomas del asma durante los tres primeros años de vida había un pronóstico benigno y que muchos de estos niños carecían de síntomas de asma a los seis años de edad. Esto está en consonancia con los resultados de este estudio, donde el $63 \%$ de los niños, diagnosticados de asma y expuesto a la medicación a los tres años, no tenía síntomas de asma en seis años de edad.

La gravedad y la medicación de los la enfermedad de asma a menudo fluctúan con el tiempo. Esta contribuye a la dificultad en el correcto diagnostico del asma en los niños pequeños (10).

El asma es una enfermedad que puede provocar en el niño una psicología especial, tensión emocional y fijación a la madre. La correcta educación y establecimiento de la cooperación de la familia ayudarán a un buen desarrollo social, médico y odontológico (13).

\section{Asma y enfermedad periodontal}

Las enfermedades periodontales que afectan a los niños y adolescentes son numerosas y pueden ser conveniente agruparlas de la siguiente manera: gingivitis; que es la forma de inicio precoz de la periodontitis, la gingivitis necrotizante/periodontitis; periodontitis del adulto incipiente; y la periodontitis asociada a enfermedades sistémicas. La forma más común en los niños es la gingivitis relacionados con la pubertad. Del mismo modo, la periodontitis de inicio precoz puede ser localizada o generalizada $(14,15)$.

Se ha conocido la enfermedad periodontal como una respuesta inflamatoria, es decir, donde ocurre una reacción a las bacterias de la placa, lo que causa una inflamación crónica, sangrado gingival, el bolsillo se hace cada vez más profundo, y en última instancia, la pérdida de hueso alveolar $(14,15)$.

$\mathrm{Al}$ igual que en el asma, la respuesta inmune es la mecanismo involucrado en la patogénesis y la progresión de la enfermedad. Dado que tanto las enfermedades periodontales y respiratorias tienen una naturaleza inflamatoria y hay muchos factores que pueden afectar a su incidencia y la gravedad, el logro de aceptables y explicable resultados con firmeza depende de una preciso diseño de estudio (16). 
La gingivitis en niños asmáticos puede ser explicada, en parte, por una alteración de la respuesta inmune y por la tendencia de respiración bucal, especialmente durante un episodio de ataque asmático y deshidratación de la mucosa alveolar durante la respiración bucal (17).

Aunque el flujo salival disminuye, la concentración de mieloperoxidasa se incrementa en pacientes asmáticos. La concentración de IgE encontrada en los tejidos gingivales es elevado en los pacientes con asma, lo cual causa destrucción periodontal (18-21).

\section{Referencias bibliográficas}

1. World health organization division of mental health. Quality of life assessment an annotated bibliography compiled by Louisa Hubanks and Willem Kuy Ken. WHO/MNH/ PSF 94.1, Geneva World Health Organization; 1994.

2. Chatkin MN, Menezes AM. [Prevalence and risk factors for asthma in schoolchildren in southern Brazil]. J Pediatr (Rio J). 2005; 81(5):411-6. Portuguese.

3. Benício MH, Ferreira MU, Cardoso MR, Konno SC, Monteiro CA. Wheezing conditions in early childhood: prevalence and risk factors in the city of São Paulo, Brazil. Bull World Health Organ. 2004; 82(7):516-22.

4. Bateman ED, Hurd SS, Barnes PJ, Bousquet J, Drazen JM, FitzGerald M, Gibson P, Ohta K, O'Byrne P, Pedersen SE, Pizzichini E, Sullivan SD, Wenzel SE, Zar HJ. Global strategy for asthma management and prevention: GINA executive summary. Eur Respir J. 2008; 31(1):143-78.

5. Thomas MS, Parolia A, Kundabala M, Vikram M. Asthma and oral health: a review. Aust Dent J. 2010; 55(2):128-33.

6. Asher MI, Montefort S, Björkstén B, Lai CK, Strachan DP, Weiland SK, Williams H; ISAAC Phase Three Study Group. Worldwide time trends in the prevalence of symptoms of asthma, allergic rhinoconjunctivitis, and eczema in childhood: ISAAC Phases One and Three repeat multicountry cross-sectional surveys. Lancet. 2006; 368(9537):733-43.

7. Deen JL, Vos T, Huttly SR, Tulloch J. Injuries and noncommunicable diseases: emerging health problems of children in developing countries. Bull World Health Organ. 1999; 77(6):518-24.

8. Neffen H, Fritscher C, Schacht FC, Levy G, Chiarella P, Soriano JB, Mechali D; AIRLA Survey Group. Asthma control in Latin America: the Asthma Insights and Reality in Latin America (AIRLA) survey. Rev Panam Salud Publica. 2005; 17(3):1917.

9. Mallol J, Cortez E, Amarales L, Sánchez I, Calvo M, Soto S, Strickler A, Kyling A, Sanhueza I, Albornoz C. Prevalencia del asma en escolares chilenos: Estudio descriptivo de 24.470 niños. ISAAC-Chile. Rev Med Chil. 2000; 128(3):279-85.

10.Global Initiative for Asthma (GINA). Global strategy for asthma management and prevention; 2009.

11. Stensson M, Wendt LK, Koch G, Nilsson M, Oldaeus G, Birkhed D. Oral health in pre-school children with asthma--followed from 3 to 6 years. Int J Paediatr Dent. 2010; 20(3):165-72.

12. Martinez FD, Wright AL, Taussig LM, Holberg CJ, Halonen M, Morgan WJ. Asthma and wheezing in the first six years of life. The Group Health Medical Associates. N Engl J Med. 1995; 332(3):133-8.

13.Bimstein E, Wilson J, Guelmann $\mathrm{M}$, Primosch RE. The relationship between oral and demographic characteristics of children with asthma. J Clin Pediatr Dent. 2006; 31(2):86-9.

14.Fireman P. Atlas de alergia e inmunología clínica. Madrid: Elsevier; 2007.

15.Kinane DF, Hodge PJ. Periodontal disease in children and adolescents: introduction and classification. Periodontol 2000. 2001; 26:7-15.

16. Yaghobee S, Paknejad M, Khorsand A. Association between asthma and periodontal disease. J Dent (Iran). 2008; 5(2):47-51.

17.Hyyppä TM, Koivikko A, Paunio KU. Studies on periodontal conditions in asthmatic children. Acta Odontol Scand. 1979; 37(1):15-20.

18.Hyyppä T. Salivary immunoglobulins in children with asthma. J Periodontal Res. 1980; 15(3):227-31.

19.Lenander-Lumikari M, Laurikainen K, Kuusisto P, Vilja P. Stimulated salivary flow rate and composition in asthmatic and non-asthmatic adults. Arch Oral Biol. 1998; 43(2):151-6.

20.Hakamima KL. Asthma and oral health. A clinical and epidemiological study. Tampere: Acta Electronica Universitatis Tamperensis; 2002.

21. Wotman S, Mercadante J, Mandel ID, Goldman RS, Denning C. The occurrence of calculus in normal children, children with cystic fibrosis, and children with asthma. J Periodontol. 1973; 44(5):278-80. 